\title{
Congenital syphilis surveillance
}

\author{
Antonella Marangoni', Alessandra Moroni ${ }^{2}$, Elisabetta Tridapalli ${ }^{3}$, Maria Grazia Capretti ${ }^{3}$, \\ Antonietta D'Antuono', Marina Biagi ${ }^{2}$, Sanzio Ruscello², Franca Savioli'2, Roberto Cevenini' \\ I DESOS, Sez. Microbiologia, Università di Bologna, \\ 2 U. O. Microbiologia, \\ 3 U. O. Neonatologia, \\ 4 U.O. Dermatologia, Policlinico S. Orsola-Malpighi, Bologna
}

Key words: Congenital syphilis, IgMWestern Blot, Laboratory diagnosis

Protocollo di sorveglianza della sifilide congenita

\section{SUMMARY}

Congenital syphilis (CS) is mainly a consequence of the lack of antenatal care and control of sexually transmitted infections. The bedrock of the prevention of CS is syphilis diagnosis by serological screening during pregnancy. Current Italian guidelines suggest that all the pregnant women should be tested in the first trimester. Due to the frequently absence of specific signs of infection at birth, laboratory tests are often the only method for a correct CS diagnosis. The aim of this study was to evaluate the usefulness of Treponema pallidum IgMWestern Blot (WB) and Polymerase Chain Reaction (PCR) on cerebrospinal fluid (CSF) as an aid in the diagnosis of CS during a prospective surveillance study carried out at St. Orsola Hospital in Bologna, Italy, from November 2000 through June 2010.

All pregnant women during pregnancy and at delivery were screened for syphilis by ARCHITECT ${ }^{\circledR}$ Syphilis TP, Abbott. Positive samples were further analysed by Treponema Pallidum Hemagglutination Test (TPHA) and Rapid Plasma Reagin (RPR) tests, Radim. An in-house Western Blot (WB) was also performed.

Infants born to syphilis seropositive mothers were enrolled in a prospective follow up. At birth, tests were performed (including IgMWB). Infants with positive RPR tests at birth born to mothers not adequately treated received also a long bone radiograph as well as a complete CSF analysis, including Veneral Disease Research Laboratori (VDRL) (Siemens Healthcare Diagnostics) and PCR testing.

All seroreactive infants received careful follow up examinations and serological testing at 0, 3,6, 9, 12 months or until the tests became negative. In this study, positive syphilis serology was noted in $15 \mathrm{I}$ pregnant women delivering in our hospital. Fifteen women had never been adequately treated, and 9 out 15 gave birth to infected newborns. All these 9 infants had positive IgMWB results on serum samples.

Two babies had characteristic long bone lesions at X-ray examination and 3 were born preterm.

These observations confirmed that antenatal syphilis screening facilitates treatment during pregnancy and offsets vertical transmission; moreover, the use of IgMWB and careful CSF examination allowed the identification and treatment of high risk newborns.

\section{INTRODUZIONE}

Nei Paesi ad elevato standard sanitario, la diagnosi e la prevenzione della sifilide congenita sono due aspetti cardini nell'ambito delle problematiche riguardanti una vecchia infezione quale è la sifilide. Al Policlinico S. Orsola di Bologna dal 2000 è sorta una stretta rete di collaborazione tra la Microbiologia, la Neonatologia e il centro di Malattie Sessualmente Trasmesse al fine di identificare i casi a rischio per sifilide congenita.

\section{MATERIALI E METODI}

Al parto tutte le donne vengono sottoposte allo screening per ricerca di anticorpi anti-Treponema pallidum; i sieri positivi vengono poi analizzati mediante TPHA, RPR ed eventualmente anche Western Blot. Il protocollo prevede che tutti i nati da madri sieropositive per lue vengano sottoposti alle analisi sierologiche (compreso il WB IgM) e che per i nati da madri non adeguatamente seguite vengano eseguiti esami su liquor cerebrospinale (compresi i test CSF VDRL e PCR) e una radiografia delle ossa lunghe (Figura I).

\section{RISULTATI}

Nel periodo di studio sono nati 165 bambini da 151 donne sieropositive per sifilide (Figura II). In Figura III sono illustrate le provenienze geografiche di queste 151 pazienti.

Centotrentasei donne erano state trattate prima del parto, e hanno partorito 150 bambini non infetti, con WB IgM negativi alla nascita e una sieronegativizzazione entro il nono mese.

Quindici donne non erano state seguite durante la

\section{Corresponding author: Antonella Marangoni}

Policlinico S. Orsola, Servizio di Microbiologia

Via Massarenti 9 - 40138 Bologna

Tel.: 05I 63645I3 - Fax: 05। 307397 
gravidanza; 6 hanno dato alla luce neonati non infetti: i test RPR erano positivi con titoli uguali a quelli materni, mentre i test WB IgM e le indagini su liquor erano negativi.

Tutti questi bambini hanno mostrato una sieronegativizzazione entro l'anno.
I rimanenti 9 nati da madri non adeguatamente seguite viceversa risultavano infetti: tutti avevano WB IgM positivo, 4 avevano test positivi su liquor, 2 mostravano lesioni alle ossa lunghe e 3 erano prematuri (Tabella 1), di cui 2 fortemente prematuri.

Neonati nati da madri con sierologia positiva per lue

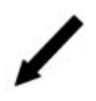

Nati da madri con trattamento effettuato prima o durante la gravidanza ( $>30 \mathrm{gg}$ prima del parto)

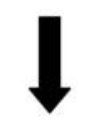

Esecuzione RPR

quantitativo, TPHA e

WB IgG/IgM su siero
Nati da madri mai trattate o con trattamento inadeguato $(<30 \mathrm{gg}$ prima del parto o con farmaci diversi dalla penicillina)

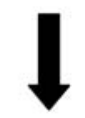

Esecuzione RPR quantitativo, TPHA e WB IgG/IgM su siero VDRL, TPHA, WB IgG/IgM, PolA PCR su liquor

$\mathrm{RX}$ ossa lunghe

TUTTI i neonati vengono inseriti nel follow up.

Secondo protocollo sono previsti prelievi seriali a 3, 6, 9, 12 mesi e/o fino a completa negativizzazione dei test treponemici

Figura I. Riassunto degli esami da richiedere sui neonati nati, rispettivamente, da madri trattate prima o durante la gravidanza per sifilide, o da madri non trattate adeguatamente durante la gravidanza.

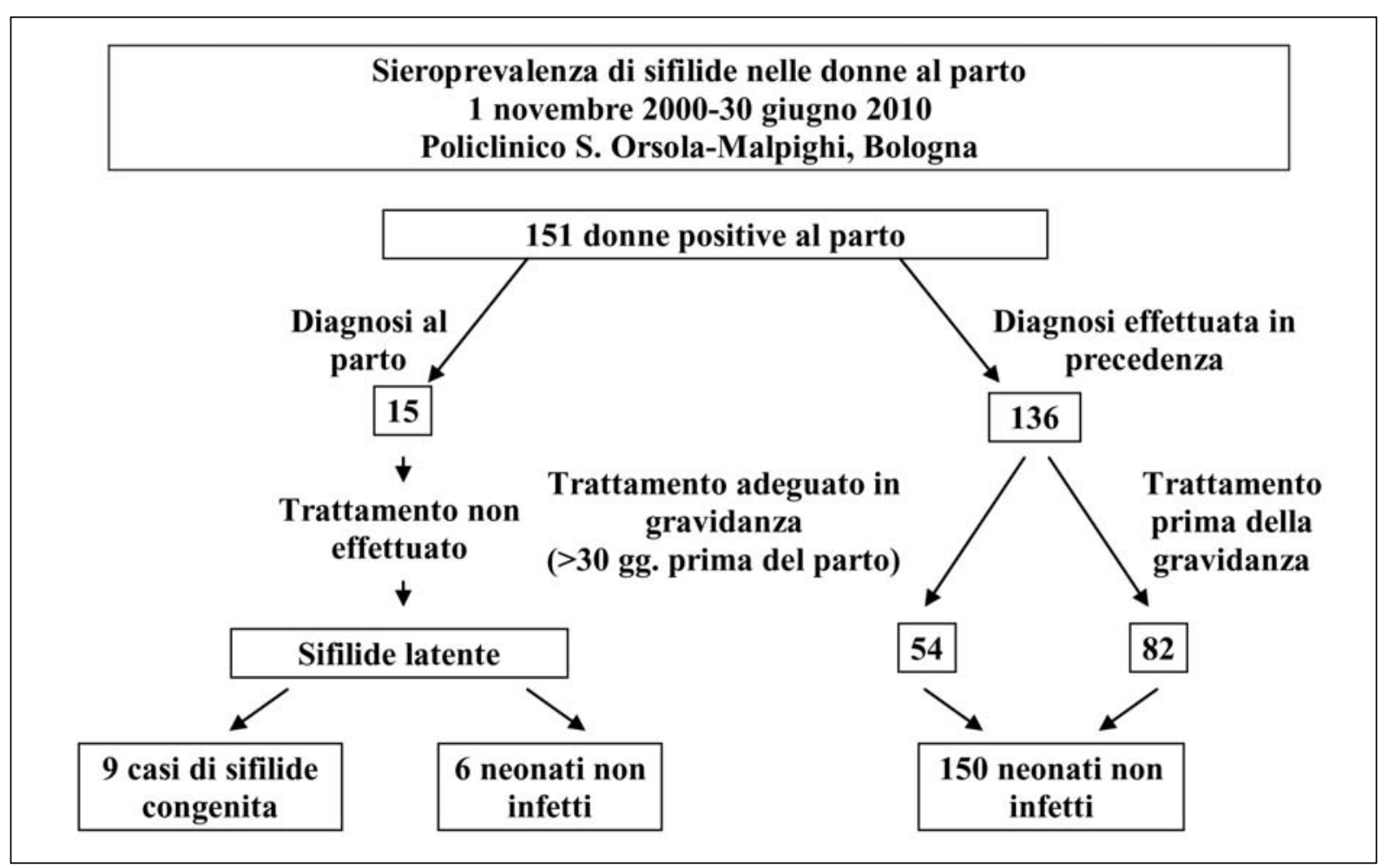

Figura II. Sieroprevalenza per sifilide nelle donne al parto nel periodo di studio (novembre 2000-giugno 2010) presso il Policlinico S. Orsola-malpighi, Bologna. 


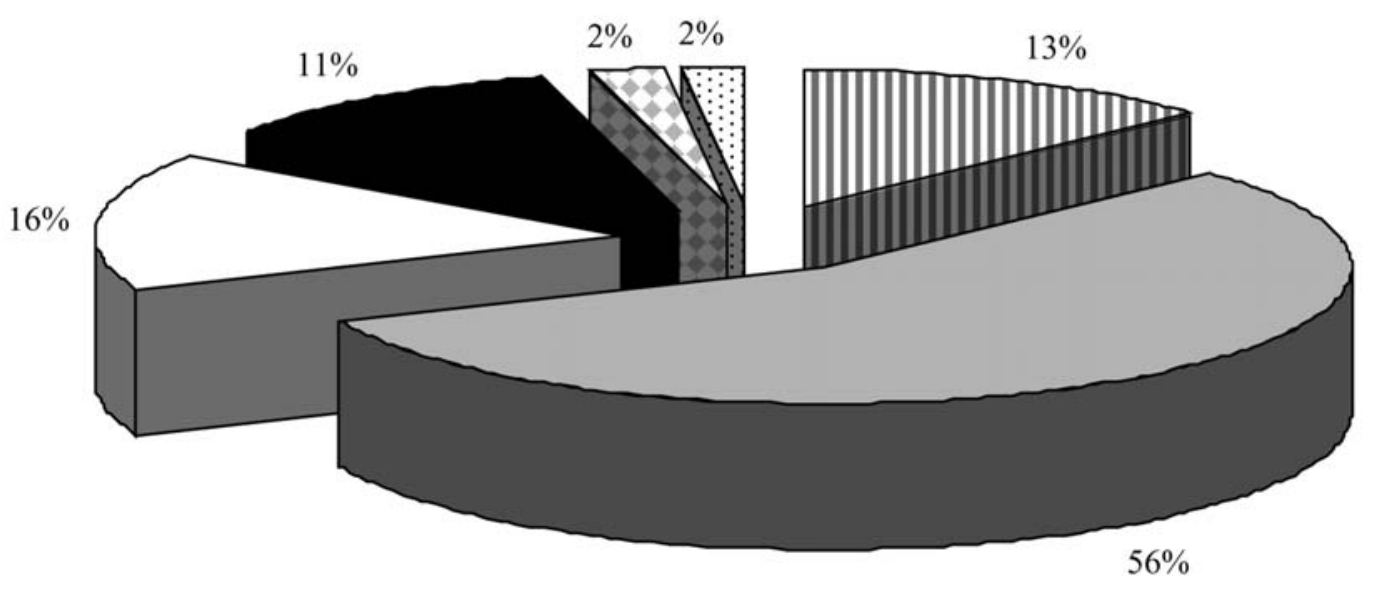

凹Italia $\square$ Est Europa $\square$ America Centro-Sud $\square$ Nord Africa $\square$ Cina $\square$ Altri

Figura III. Provenienza geografica delle donne sieropositive per sifilide ricoverate nei reparti di Ostetricia del Policlinico S. Orsola-Malpighi, Bologna.

Tabella I. Caratteristiche dei 9 neonati trovati affetti da sifilide congenita nel periodo di studio.

Profilo materno Caratteristiche dei neonati alla nascita

\begin{tabular}{|c|c|c|c|c|c|c|c|c|c|c|c|c|}
\hline & & & & & & alla na & scita & & & all'ultin & no foll & w-up \\
\hline & $\begin{array}{c}\text { Paese di } \\
\text { provenienza }\end{array}$ & $\begin{array}{l}\text { Stato } \\
\text { civile }\end{array}$ & $\begin{array}{c}\text { Età gestaz. } \\
\text { (settim.) }\end{array}$ & $\begin{array}{l}\text { Peso } \\
\text { (gr.) }\end{array}$ & $\begin{array}{l}\text { IgM } \\
\text { WB }\end{array}$ & $\begin{array}{r}\text { RPR } \\
\text { CSF }\end{array}$ & $\begin{array}{l}\text { VDRL } \\
\text { PCR }\end{array}$ & PolA & $\begin{array}{c}\text { RX } \\
\text { Ossa lunghe }\end{array}$ & $\begin{array}{c}\text { Età } \\
\text { (mesi) }\end{array}$ & RPR & PHA \\
\hline Caso I & I Romania & Nubile & 24 & 695 & + & + & ND & ND & normale & 12 & - & + \\
\hline Caso 2 & 2 Romania & Coniugata & 26 & 495 & + & + & ND & ND & normale & 12 & - & + \\
\hline Caso 3 & 3 Romania & Nubile & 34 & 2150 & + & + & - & ND & normale & 4 & - & + \\
\hline Caso 4 & Russia & Coniugata & 40 & 3660 & + & + & + & ND & normale & 12 & - & + \\
\hline Caso 5 & 5 Ucraina & Nubile & 39 & 3250 & + & + & + & + & normale & 24 & - & + \\
\hline Caso 6 & 6 Romania & Coniugata & 38 & 2800 & + & + & - & ND & anomalo & 12 & - & + \\
\hline Caso 7 & 7 Romania & Nubile & 38 & 3800 & + & + & + & + & normale & 12 & - & + \\
\hline Caso 8 & 8 Romania & Nubile & 36 & 3000 & + & + & + & + & anomalo & 12 & $+/-$ & + \\
\hline Caso 9 & 9 Romania & Nubile & 38 & 3000 & + & + & - & - & anomalo & 12 & - & + \\
\hline
\end{tabular}

\section{DISCUSSIONE}

Lo screening sierologico per sifilide durante il primo trimestre dovrebbe essere effettuato da parte di tutte le donne (D.M. 10/09/1998), per identificare i casi positivi ed iniziare un'adeguata terapia antibiotica. I ginecologi dovrebbero quindi prestare maggior attenzione al problema, soprattutto per le pazienti di origine straniera e in particolare per quelle provenienti dai Paesi dell'Est Europa (1-2).

Come si evidenzia dai dati di questo studio e dai precedenti (2-3), lo screening per sifilide effettuato sulle madri al momento del parto permette di individuare immediatamente i neonati a rischio.

Sui neonati è particolarmente utile un'accurata diagnosi di laboratorio per l'identificazione dei neonati infetti: solo questi verranno sottoposti a terapia antibiotica, risparmiando così un inutile trattamento e conseguente ospedalizzazione ai neonati non infetti (1).

\section{BIBLIOGRAFIA}

1. Marangoni A, Moroni A, Tridapalli E, et al. Antenatal syphilis serology in pregnant women and follow-up of their infants in northern Italy. Clin Microbiol Infect 2008; 14: 1065-8.

2. Tikhonova L, Salakhov E, Southwick K, et al. Congenital syphilis in the Russian Federation: magnitude, determinants, and consequences. Sex Transm Infect 2003; 79: 106-10.

3. Tridapalli E, Capretti MG, Sambri V, et al. Prenatal syphilis infection is a possible cause of preterm delivery among immigrant women from Eastern Europe. Sex Trans Infect 2007; 83: 102-5. 